\title{
Quality of life and associated factors after surgical treatment of vulvar cancer by vulvar field resection (VFR)
}

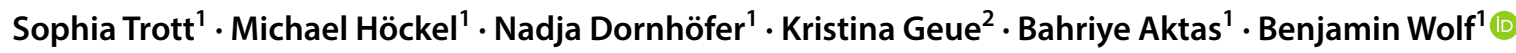

Received: 23 September 2019 / Accepted: 5 May 2020 / Published online: 19 May 2020

(c) The Author(s) 2020

\begin{abstract}
Purpose To investigate patient-reported quality of life $(\mathrm{QoL})$ and associated factors in vulvar cancer patients treated surgically by vulvar field resection (VFR) without adjuvant radiation.

Methods We retrospectively evaluated patient-reported QoL as part of the prospective monocentric VFR trial using the 30-item European Organization for Research and Treatment of Cancer quality-of-life questionnaire (EORTC QLQ-C30) supplemented by a question assessing sexual activity. All patients had been treated by VFR and no participant had received adjuvant radiotherapy. The gynecologic cancer lymphedema questionnaire (GCLQ) was used to determine the presence of lymphedema. Structured telephone interviews were conducted to assess postoperative sequelae and long-term complications. Results Forty-three VFR patients (median age 63 years) were available for QoL assessment. Thirty-eight (88\%) had received inguinal lymph-node dissection in addition to VFR. Mean global QoL (global health status) rating among all patients was $66.1( \pm 25.5)$ on a scale from 0 to 100 with higher scores indicating better QoL. Higher GCLQ scores were significantly associated with lower global QoL scores (Spearman's rank correlation $\rho=-0.7, p<0.0001$ ). The presence of preoperative co-morbidities and postoperative wound-healing complications were also linked to reduced QoL ( $p<0.01$ for both). In a multivariable regression model, there was a significant interaction between preoperative co-morbidities and wound-healing complications with regard to global QoL $(p<0.05)$.

Conclusion Overall, VFR patients exhibit good quality of life postoperatively. The presence of lymphedema, wound-healing complications, and preoperative morbidities were associated with reduced QoL. Prospective longitudinal studies have to confirm our findings in the future.
\end{abstract}

Keywords Quality of Life $\cdot$ Vulvar cancer $\cdot$ Vulvar field resection $\cdot$ Morbidity $\cdot$ Lymphedema $\cdot$ Wound healing complications

\section{Introduction}

Vulvar cancer accounts for about $5 \%$ of all malignancies of the female genital tract $[1,2]$. Even though it is an uncommon cancer, its incidence has been increasing in recent

Electronic supplementary material The online version of this article (https://doi.org/10.1007/s00404-020-05584-5) contains supplementary material, which is available to authorized users.

Benjamin Wolf

benjamin.wolf@medizin.uni-leipzig.de

1 Department of Gynecology, University Hospital Leipzig, Liebigstr. 20a, 04103 Leipzig, Germany

2 Division of Medical Psychology and Medical Sociology, University of Leipzig, Leipzig, Germany decades [3, 4]. Whereas vulvar cancer is still regarded as a tumor of the elderly, the rising incidence has been driven largely by new cases among younger women $[4,5]$. According to the Surveillance, Epidemiology, and End Results (SEER) database, $59 \%$ of patients have localized and another $29 \%$ regional disease at the time of diagnosis with $86.3 \%$ and $52.6 \%$ surviving for 5 or more years in each group, respectively [6]. Therefore, as most patients with vulvar cancer survive the disease, the number of years that patients live with treatment-related long-term complications and sequelae is increasing and post-treatment quality of life (QoL) deserves special consideration when counseling affected women about their treatment options. We have proposed vulvar field resection (VFR) as a novel approach to the surgical treatment of vulvar cancer [7, 8]. VFR is based on the theory of ontogenetic cancer fields which holds that malignant tumors 
infiltrate specific ontogenetically determined tissue domains in a stepwise and predictable manner. VFR is characterized by the local resection of a tumor within its ontogenetically specified field of potential growth (cancer field). While in some anatomic regions, this approach necessitates resection margins wider than $8 \mathrm{~mm}$, other tissues directly abutting the tumor but belonging to a different ontogenetic domain can be preserved as they are not at risk for tumor involvement even when they are in close spatial proximity $(<8 \mathrm{~mm})$. This facilitates optimal anatomic reconstruction by sparing important flap-donation tissues such as the labia majora in most cases and minimizes morbidity. Regional assessment for lymphatic tumor spread and therapeutic lymph-node dissection is another integral part of VFR. Adjuvant radiation is not administered either to the tumor field or the lymphatic drainage regions after VFR. We have already demonstrated excellent surgical and oncological outcomes achieved in the monocentric prospective Leipzig VFR trial [8]. In this trial, 97 consecutive patients were included of which $40 \%$ had lymph-node metastases and 36\% had stage II disease or higher. Progression-free survival after 36 months was $85.1 \%$ and disease-specific survival was $86.0 \%$. Here, we now describe patient-reported outcome measures evaluating QoL. Especially in light of the good survival outcomes, QoL is of great clinical importance and possibly more relevant than objective functional results after surgery. Because lymphedema of the lower extremities is one of the most prevalent and disturbing sequelae in vulvar cancer survivors, we here report its presence in VFR patients and investigate its correlation with QoL.

\section{Methods}

\section{Study design}

We performed a retrospective post hoc QoL analysis in patients who participated in the Leipzig School VFR study. The study was a prospective monocentric observational trial designed to evaluate the feasibility and surgical safety of VFR at the University of Leipzig's cancer center [8]. Patients were eligible for participation if they were 18 years or older, had ontogenetic stage $1-3 b$ vulvar cancer with or without lymph-node involvement, comprising FIGO stages I-III (more information regarding ontogenetic staging is available in the supplementary online resource p. 1 and Table S1 on p. 3) and had not undergone previous surgical or radiation therapy of the vulva. In addition, all patients were seen preoperatively by an anesthesiologist and had to be deemed fit for the operation. All patients provided written informed consent. Ethical approval was granted by the Leipzig University Institutional Review Board (156-2009-06072009 and 120-12-16042012). The trial (which was transformed into a multicentric study in February 2019) is registered at the German clinical trials registry (DRKS00013358). According to the above criteria, 97 consecutive vulvar cancer patients were included in the study between March 1, 2009, and June 8, 2017, the detailed surgical and oncological characteristics of this cohort have been published elsewhere [8]. A subset of this group consisting of all patients who were still alive in July 2017 and who were willing and capable to participate in QoL assessment as outlined below was included in the present investigation (Figure S1 in the supplementary online resource p. 4).

\section{Clinical and pathological data acquisition}

All clinical and pathological data referred to in this investigation were collected prospectively as part of the Leipzig School VFR study. Medical co-morbidities were also assessed preoperatively by a gynecologic oncologist and an anesthesiologist as part of the study. All relevant information for the present investigation was later retrieved from the study records.

\section{Structured telephone interviews}

All of the 94 patients who were still alive in July 2017 were contacted via telephone and submitted to a structured interview concerning long-term sequelae (including vulvar and perineal dysesthesia, problems with micturition and defecation, impaired pliability of the introitus, and subjective perception of vulvar symmetry). The complete structured interview questions are available in the supplementary online resource on p. 5.

\section{Quality-of-life assessment}

Once a patient had completed the telephone interview and was physically and mentally capable of participating in QoL assessment, she was asked to complete the 30-item European Organization for Research and Treatment of Cancer Quality of Life Questionnaire (EORTC QLQ-C30) [9] which is currently one of the most commonly used and best validated instruments for patient-reported QoL assessment [10]. This questionnaire comprises five multi-item function scales (physical, role, emotional, cognitive, and social); three multi-item symptom scales (fatigue, nausea, and pain); six single-item symptom scales (dyspnea, insomnia, appetite loss, constipation, diarrhea, and financial difficulties); and one multi-item global QoL scale [9]. All raw scores are linearly transformed into a scale ranging from 0 to 100 with 
high values representing a higher functional level (EORTC QLQ-C30 functional scales) and high levels on symptom scales indicating the presence of more severe symptoms. Good-to-high reliability (Cronbach's alpha $>0.70$ ) and good construct validity have been demonstrated for all scales of the German version [11].

\section{Patient-reported lymphedema assessment}

We assessed the patient-reported presence of lymphedema using a German translation of the gynecologic cancer lymphedema questionnaire (GCLQ) [12]. The GCLQ is a 20-item questionnaire which evaluates the presence of patient-reported lower leg symptoms frequently associated with lymphedema (heaviness, swelling, presence of infections, aching, numbness, and physical functioning) during the past 4 weeks. Each of the 20 items is scored 1 or 0 and the total score is calculated by summation of all 20 items. Higher scores, therefore, indicate the presence of more severe lymphedema associated symptoms. Excellent internal consistency reliability has been reported for the English version of the questionnaire (Cronbach's alpha $=0.95$ ) [12].

\section{Assessment of sexual activity}

In addition to the EORTC QLQ-C30 and the GCLQ, we asked patients whether they were sexually active using selfdeveloped questionnaire items. If they were not engaging in sexual activity, we asked them to further specify the reason for their sexual abstinence using the following categories: lack of sexual partner, relationship problems, consequence of VFR treatment, or unspecified reasons which the patients did not want to disclose.

\section{Statistical analysis}

All data were gathered and processed using Microsoft Excel (2016). Scoring of EORTC QLQ-C30 questionnaires was done in accordance with the current edition of the scoring manual [13]. Missing values were handled as outlined in the questionnaire guidelines. Scales were only analyzed when at least half of all relevant questions had been answered. In these cases, the mean of the answer of all items of the relevant scale was used as a substitute for missing values. For further statistical analysis, R [14] was used. We used non-parametric tests (Wilcoxon rank-sum test, Kruskal-Wallis test, and Chi-square test) to determine intergroup differences. Spearman's rank correlation was used to calculate associations between GCLQ and EORTC QLQ-C30 scores. Categorical characteristics are reported as percentages, while medians and IQR are given for quantitative data. An exception to this is the reporting of EORTC QLQ-C30 scores when they are not subjected to further statistical testing. This is to facilitate the comparison with QoL data published elsewhere which is generally given as mean and standard deviation. We used linear regression analysis as implemented in the $\mathrm{glm}$ function of R [14] to investigate the interaction of preoperative co-morbidities and postoperative wound-healing complications in their association with global QoL.

\section{Results}

The patient selection process is outlined in figure $\mathrm{S} 1$ of the supplementary online resource (p. 4). Of the 64 patients who could be contacted, 45 completed the questionnaire package as outlined in the methods section, yielding a response rate of $70.4 \%$. Two patients had to be excluded from the study because they had received postoperative radiotherapy thus violating the study protocol (both patients had followed the recommendations of their general gynecologists who were providing follow-up care). Therefore, 43 patients were included in the final analysis. Forty-one EORTC QLQ-C30 forms were fully completed, while missing values had to be imputed in two cases as outlined in the methods section (one patient did not report on appetite and another omitted a question concerning role function). The median time elapsed between operation and patient assessment (follow-up) was 44 (IQR 25.7-69.7) months. Basic sociographic and clinical information are compiled in Table 1. The median age of our cohort was 63 years (IQR 56-75). Co-morbidities were present in 29 patients $(67 \%)$. There were many advanced cases represented by the presence of nodal involvement in ten patients (23\%), a median tumor size of $15 \mathrm{~mm}$, and FIGO disease stage II or higher in nine patients $(22 \%)$.

\section{Quality of life}

The mean global QoL score was $66.1( \pm 26.8)$. Of all EORTC QLQ-C30 function scales assessed, mean role functioning was lowest with $68.3( \pm 33.9)$ points, while mean cognitive functioning was highest with $80.6( \pm 26.7)$ points. Out of the three multi-item symptom scales, fatigue was on average rated highest with $34.8( \pm 31.1)$ points, and out of the six single-item symptom scales evaluated with the EORTC QLQ-C30, sleep disturbance was highest with 28.7 ( \pm 36.8 ) points (Table 2$)$. There was a significant association between decreased global QoL with increasing patient age at the time of operation $(\rho=-0.3, p=0.048)$. As determined by a Wilcoxon rank-sum test, the presence of preoperative co-morbidities and the occurrence of postoperative wound-healing complications were associated with significantly reduced global QoL $(p=0.0003$ and 
Table 1 Sociographic, patient, tumor, and treatment characteristics
Patients $(n=43)$

Socio-demographic characteristics (at the time of QoL assessment)

Age, years (median, IQR)

63

$56-75$

Marital status-n, \%

Single

Married

Widowed

Separated

Educational status-n, \%

None

Primary

Secondary

Tertiary

\section{2}

22

12

7

51

28

Patient characteristics (at the time of QoL assessment)

Body mass index, $\mathrm{kg} / \mathrm{m}^{2}$ (median, IQR)

American Society of Anesthesiologists (ASA) score-n, \%

1

2

3

$3 \quad 7$

$20 \quad 47$

$13 \quad 30$

7

30

Comorbidities or risk factors-n, $\%$

Hypertension

Diabetes mellitus

Heavy smoking

Dementia or depression

Previous malignancies

None

15

25

Tumor characteristics

Histological tumor type-n, \%

Squamous cell

Basal cell

Tumor size in mm-median, IQR

Maximum diameter

10-34

Infiltration depth

3-12

Pathological tumor stage-n, \%

1a

12

$1 b$

33

77

2

Ontogenetic tumor stage (oT)- n, \%

1

2

$3 a / 3 b$

Pathological node stage-n, $\%$

0

1a

$1 \mathrm{~b}$

$2 \mathrm{~b}$

$2 \mathrm{c}$

$\begin{array}{ll}28 & 65 \\ 9 & 21 \\ 6 & 14\end{array}$

$33 \quad 77$

25

37

12

4

9

International Federation of Gynecology and Obstetrics (FIGO) stage-n, \%

IA

5

28

12

IB

65

II

IIIA 
Table 1 (continued)

\begin{tabular}{|c|c|c|}
\hline \multicolumn{3}{|l|}{ Patients $(n=43)$} \\
\hline IIIB & 1 & 2 \\
\hline IIIC & 4 & 9 \\
\hline \multicolumn{3}{|l|}{ Treatment characteristics } \\
\hline \multicolumn{3}{|c|}{ Type of lymph-node dissection-n, $\%$} \\
\hline None & 5 & 12 \\
\hline Sentinel & 7 & 16 \\
\hline First-line inguinal & 9 & 21 \\
\hline Total inguinal $^{\mathrm{a}}$ & 22 & 51 \\
\hline \multicolumn{3}{|c|}{ Type of anatomic reconstruction-n, $\%$} \\
\hline None (direct closure) & 4 & 9 \\
\hline Random flaps & 24 & 56 \\
\hline Axial pattern flaps & 15 & 35 \\
\hline \multicolumn{3}{|c|}{ Selected complications/sequelae } \\
\hline \multicolumn{3}{|c|}{ Postoperative inguinal wound infection leading to wound breakdown, stratified for nodal status-n, $\%$} \\
\hline $\mathrm{pN} 0, \mathrm{n}=33$ & 4 & 12.2 \\
\hline $\mathrm{pN} 1, \mathrm{n}=10$ & 4 & 40 \\
\hline \multicolumn{3}{|c|}{ Occurrence of postoperative lymphedema according to LNE type ${ }^{b}-n, \%$} \\
\hline No LNE & 1 & 20 \\
\hline Sentinel LNE & 2 & 28.6 \\
\hline First-line inguinal LNE & 4 & 44.4 \\
\hline Total inguinal LNE & 10 & 45.5 \\
\hline
\end{tabular}

$I Q R$ : interquartile range

aincluding two patients with distal pelvic LND and one patient with inguinopelvic (lacunar) LND

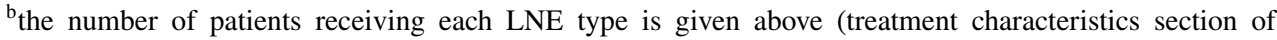

Table 1). See also Fig. S3A

Table 2 Results from QoL assessment with EORTC QLQ-C30

\begin{tabular}{lcl}
\hline & Mean & $\begin{array}{l}\text { Standard } \\
\text { deviation }\end{array}$ \\
\hline Multi-item function scales & & \\
Physical functioning & 73.5 & 25.6 \\
Role functioning & 68.3 & 33.9 \\
Emotional functioning & 75.9 & 27.8 \\
Cognitive functioning & 80.6 & 26.7 \\
Social functioning & 71.7 & 32.0 \\
Multi-item symptom scales & & \\
Fatigue & 34.8 & 31.1 \\
Nausea and vomiting & 4.3 & 13.2 \\
Pain & 33.7 & 35.5 \\
Single-item symptom scales & & \\
Dyspnea & 18.6 & 29.4 \\
Sleep disturbance & 28.7 & 36.8 \\
Appetite loss & 8.7 & 19.6 \\
Constipation & 11.6 & 25.1 \\
Diarrhea & 9.3 & 23.4 \\
Financial impact & 14.7 & 27.5 \\
Multi-item QoL & & \\
Global quality of life (global health & 66.1 & 25.5 \\
$\quad$ status) & & \\
\hline
\end{tabular}

$p=0.001$, respectively, Fig. 1a, b). Multivariable linear regression analysis investigating the effect of preoperative co-morbidities and wound-healing complications on global QoL revealed a significant interaction between the two factors $(p<0.05$, Table S2 in the supplementary online resource p. 4). The presence of overweight or obesity [body mass index (BMI) $\geq 25 \mathrm{~kg} / \mathrm{m}^{2}$ ] was not linked to global QoL differences $(p=0.36$,). The amount of time elapsed between operation and EORTC QLQ-C30 completion ( $<48$ months vs. $\geq 48$ months) was also not associated with changes in $\operatorname{QoL}(p=0.74)$. As determined by Kruskal-Wallis tests, none of the sequelae assessed in the structured telephone interview were linked to significantly reduced QoL (Figure S2 in the supplementary online resource p. 6).

\section{Lymphedema}

The mean GCLQ score among all 43 patients in our study was 4.7 ( \pm 4.7$)$. Using a GCLQ score of $\geq 5$ as cut-off value (yielding a positive and negative predictive value for the presence of lymphedema of $88.9 \%$ and $87 \%$, respectively [12]), lymphedema was present in a total of 17 women 


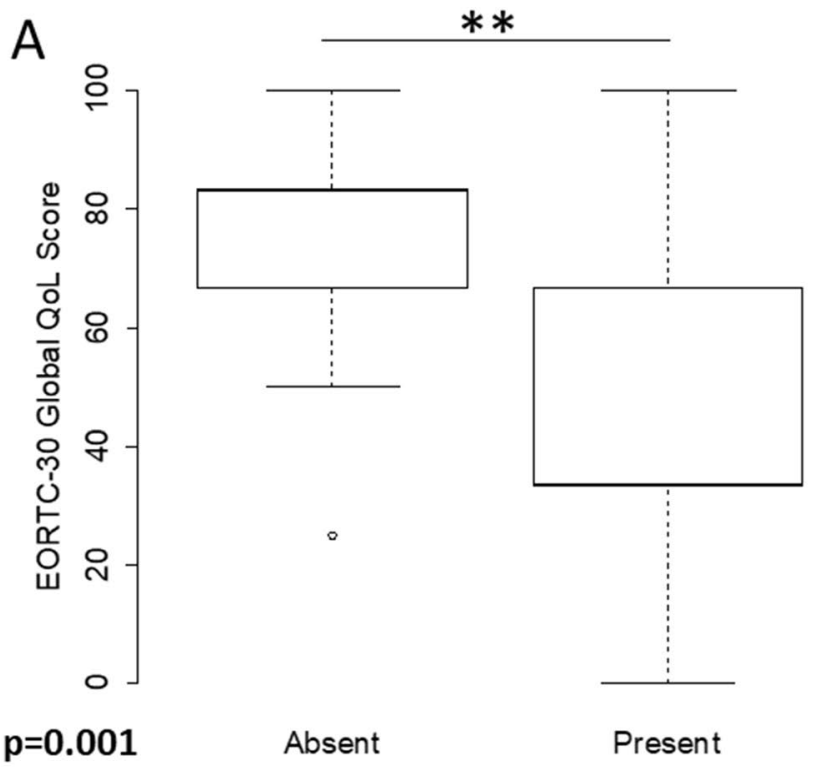

Postoperative wound break down

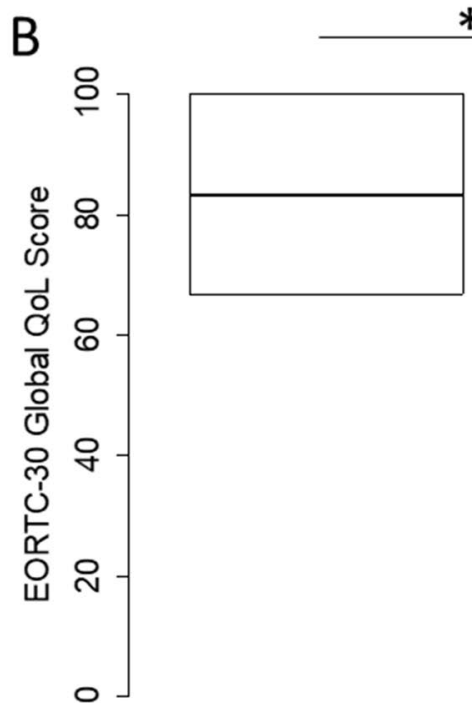

$\mathrm{p}=0.0003$
**

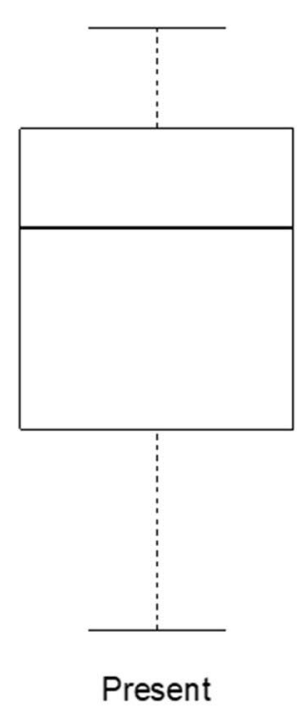

Preoperative morbidities

Fig. 1 Factors associated with QoL. Boxplots indicating the distribution of global QoL scores stratified for the occurrence of postoperative wound breakdown (a) and the presence of preoperative morbidities as outlined in Table 1 (b)

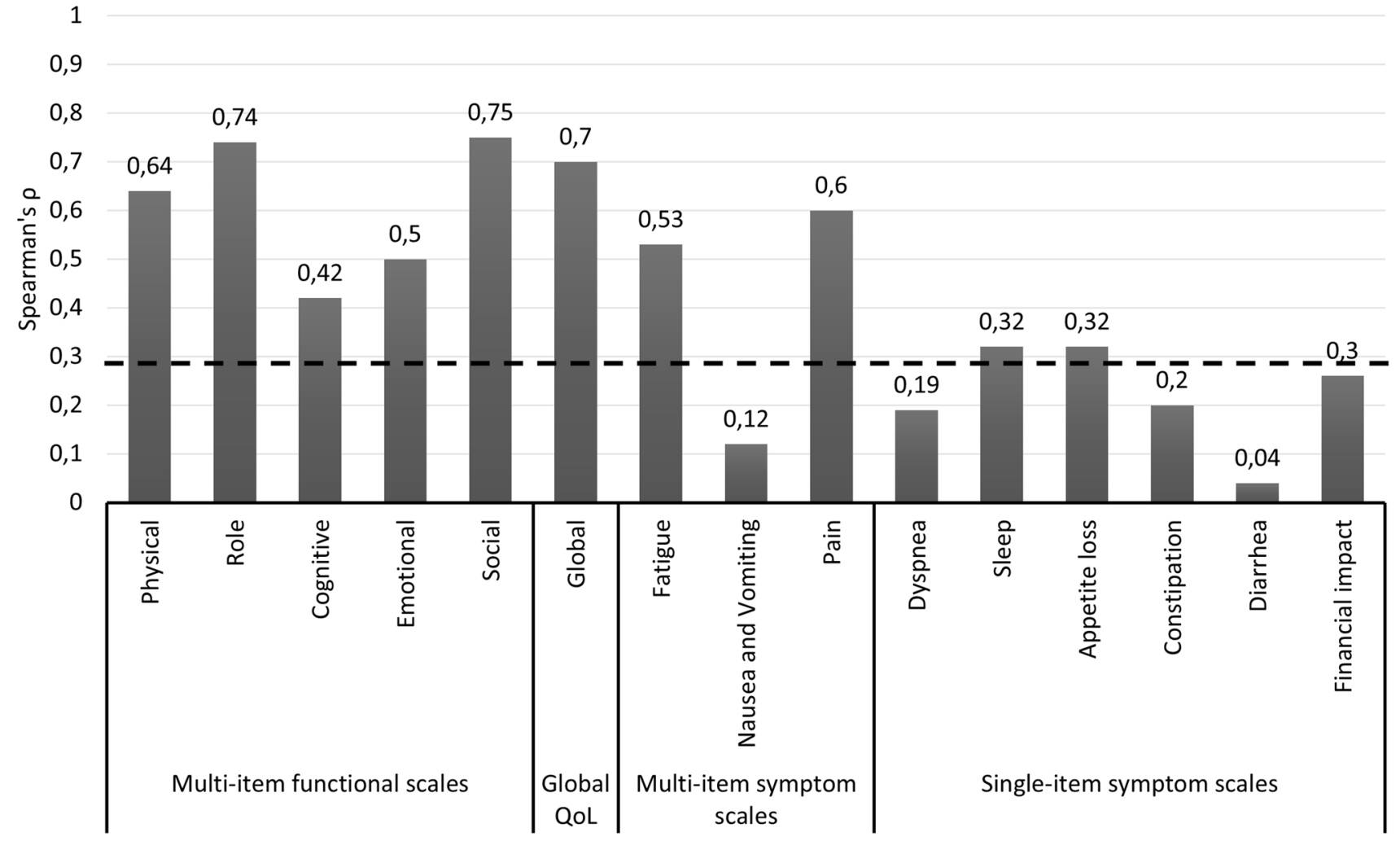

Fig. 2 Correlation between GCLQ score and QoL. Results from Spearman's correlation test evaluating the relationship between the scores of the GCLQ and the EORTC-QLQ-C30 scales. The dashed line indicates the significance threshold of $p=0.05$. Higher $\rho$ values indicate a stronger association 


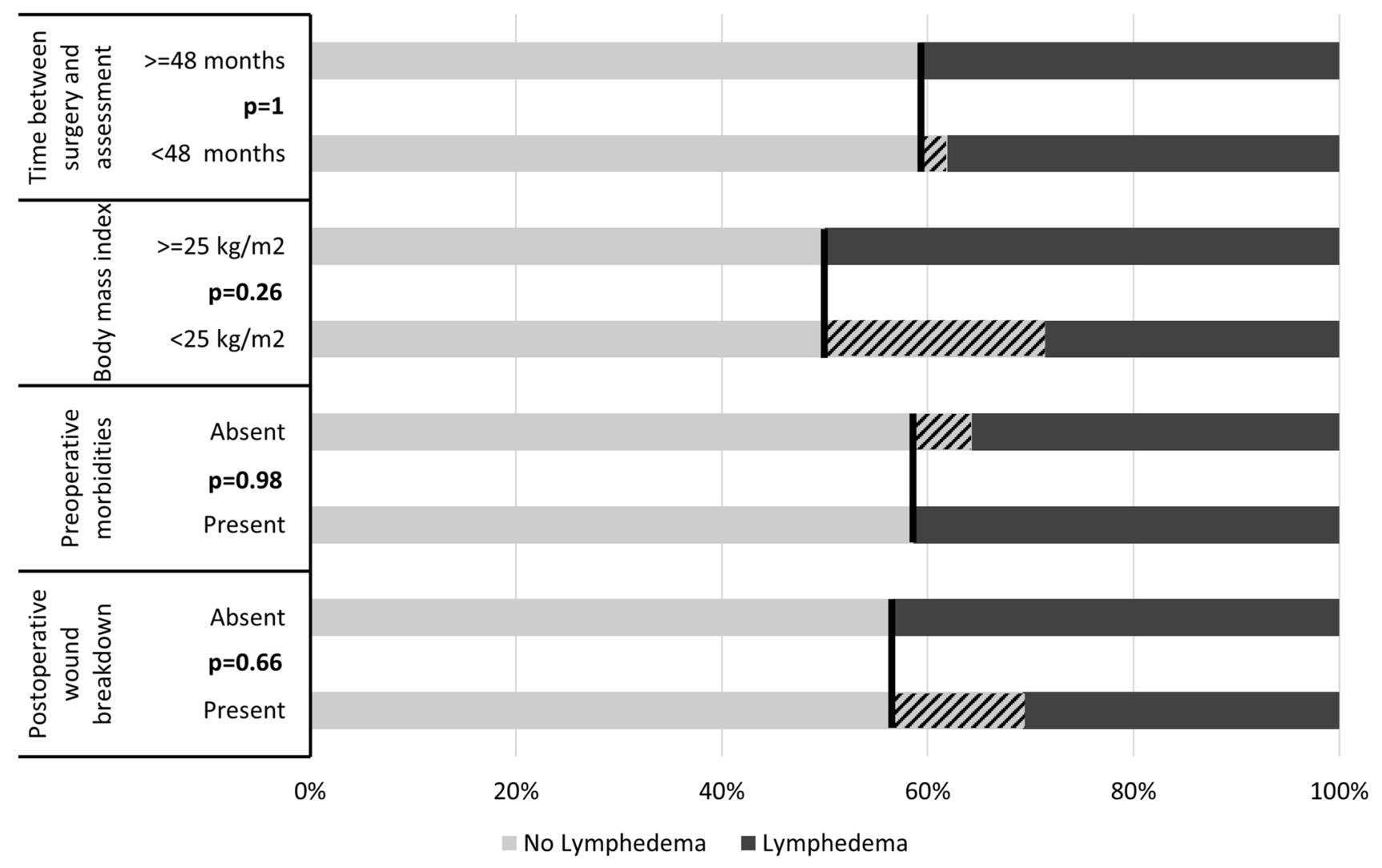

Fig. 3 Factors associated with lymphedema. Barplots indicating the percentage of patients with lymphedema as determined by a GCLQ score of $\geq 5$, stratified for different risk factors. The shaded plot segments highlight the size of the intergroup differences

(39.5\%). Higher GCLQ scores were significantly associated with reduced QoL represented by worse outcomes on all five functional subscales (Fig. 2). Neither the presence of preoperative morbidities, a BMI of $\geq 25 \mathrm{mg} / \mathrm{kg}^{2}$, postoperative wound breakdown, nor a longer time since the operation ( $\geq 48$ months) were significantly associated with changes in lymphedema symptoms as determined by Chi-square tests (Fig. 3).

\section{Sexual activity}

Only 8 (18.6\%) out of 43 patients reported to be sexually active and three patients did not disclose any information regarding sex life. Among the remaining 32 patients, the most common reason for sexual inactivity was the absence of a sexual partner (43.8\%). Nine women $(28.1 \%)$ reported that sexual activity was prohibited by sequelae of VFR. Six patients attributed their sexual inactivity to relationship problems unrelated to VFR (including medical problems of their partner) and three patients did not further specify the reason for their sexual abstinence. Among the 40 patients with available information, sexual activity correlated inversely with patient age and positively with global QoL
(Wilcoxon rank-sum test, $p=0.047$ and $p=0.029$, respectively, Fig. 4).

\section{Anatomical reconstruction}

All but four patients (9\%) received anatomical reconstruction using local flaps (Table 1). The type of reconstruction used (direct closure, axial-pattern, or random-pattern flaps) was not related to postoperative QoL (Kruskal-Wallis test, $p=0.4345$, Figure $\mathrm{S} 4$ in the supplementary online resource p. 7).

\section{Discussion}

In this study, we present for the first time results from QoL assessment in patients who underwent VFR with therapeutic lymph-node dissection and anatomical reconstruction. As VFR exhibits excellent cure rates [8], postoperative QoL is of great relevance. In several aspects, QoL in our patients was not different from that in a representative German reference population of women without cancer. For example, most of our study patients were 60 years of age 

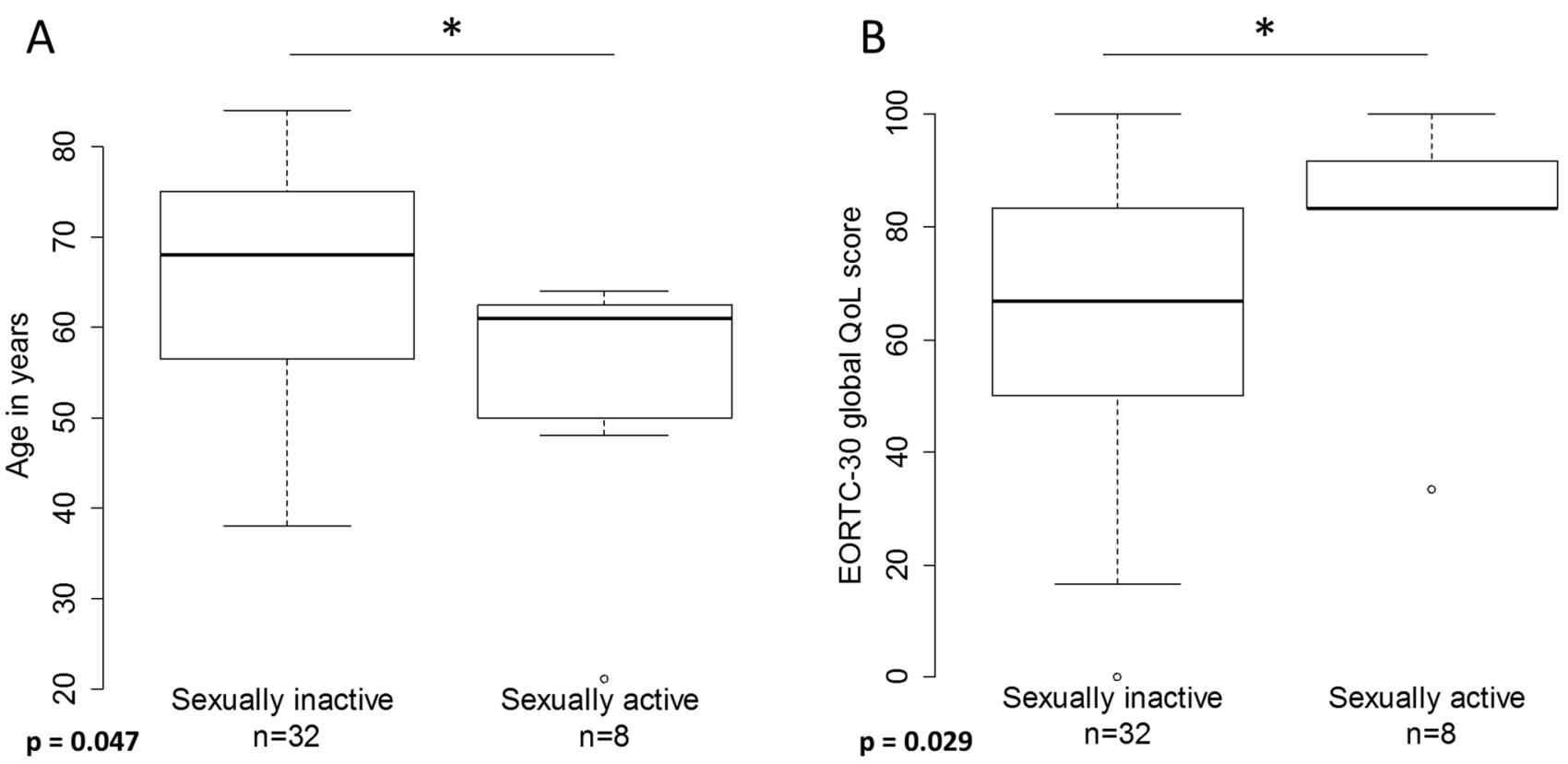

Fig. 4 Correlation of global QoL with age and sexual activity. Boxplots indicating the age distribution of sexually active and inactive patients (a) and the distribution of global QoL scores among the same cohorts (b). Three patients did not disclose any information regarding sexual activity

or older $(n=28,65 \%)$. Even though we observed a statistically significant decline in global QoL status with increasing patient age, mean global QoL in the subgroup of patients aged $\geq 60$ years was still $63.5( \pm 24.2)$. In the German reference population of healthy women [15], the mean global QoL score was 69.0 for subjects aged 60-69 years and 60.2 for women aged more than 70 years.

Comparison of our QoL data with that from other studies involving vulvar cancer patients is limited because of different patient selection criteria, and varying study protocols (e.g., time of QoL assessment), and small-sample sizes impeding subgroup analyses. Keeping these limitations in mind, nevertheless, a cautious comparison with some studies can be made. For example, Hellinga et al. [16] report a mean global QoL score of 75.76 in a group of 22 patients who were treated for (pre-)malignant lesions of the vulvoperineal area surgically and who underwent reconstruction using a lotus petal flap. In their cohort, however, the patients appeared to be somewhat healthier at the outset of treatment as suggested by the absence of any patient with an ASA score higher than 2. In contrast, $28 \%$ of the patients in our group were scored ASA 3, and our data demonstrate that preoperative morbidities are associated with reduced postoperative QoL. Moreover, 54\% of the patients in their study were not treated for vulvar cancer but for other disease entities. In another study, Novackova et al. [17] compared QoL in vulvar cancer patients treated surgically with SLNB (CON group) or with inguinofemoral lymphadenectomy (RAD group). Global QoL was 70.5 and 72.5 after 6 and 12 months, respectively, in the SLNB group, while it was
64 and 65 after 6 and 12 months in the inguinofemoral lymphadenectomy group. Additionally, 50\% of the RAD group received radiotherapy due to groin node metastasis. The QoL of the RAD group was only slightly worse in comparison to our findings. This might be attributable to the median number of lymph nodes removed per groin. The median number in the RAD group was 4.7 (range 3-6), whereas it was 8 (range 0-17) in our study. Also, there was no information regarding whether the RAD group was treated with local excision or modified radical vulvectomy. These differences in treatment and extent of (inguinal) and vulvar surgery clearly impede comparison.

An important result was the clear association between the preoperative presence of medical co-morbidities and reduced postoperative QoL ( $p=0.0003$, Fig. 1b). Even though this is not unexpected, it is of special relevance in our study as it can serve as a proxy for preoperative QoL. For example, arterial hypertension [18] and diabetes mellitus [19] have both been shown to negatively impact QoL in large studies. Therefore, it is not unlikely that a significant number of patients with low EORTC QLQ-C30 scores postoperatively had already been suffering from lower QoL before surgery.

Importantly, we found that wound-healing complications were also associated with reduced global QoL, with a significant interaction observed with the presence of preoperative co-morbidities in a multivariable linear regression model. The decreased postoperative global QoL in patients who had wound-healing complications is, therefore, probably best explained by their preoperative constitution rather than by immediate effects of secondary wound healing. This is in 
accord with our previous findings, indicating that long-term functional and cosmetic outcomes are not inferior in patients who experience wound-healing complications as compared to those who do not have such problems [8]. We did not observe an effect of the time interval elapsed since surgery and quality of life. Interestingly, a recent longitudinal study observed a significant decrease in several QoL scales [20]. In that study, almost $30 \%$ of patients received adjuvant radiation and the authors attributed the worsening QoL at least partially to side effects of radiotherapy. VFR does not necessitate adjuvant radiation even in the presence of risk factors and this clear advantage over the conventional therapy might contribute to stable QoL postoperatively. There was also no association between various postoperative sequelae assessed in the structured interviews and QoL (Figure S2 in the supplementary online resource p. 6). This finding is probably best explained by the so-called "response shift" observed in QoL investigations involving cancer patients. This concept describes how QoL in cancer survivors is better than expected in light of treatment sequelae and long-term symptoms, because these patients experience a change in their frame of reference thereby reconceptualizing, reprioritizing, and recalibrating different psychological and physical aspects contributing to overall QoL [21, 22].

The presence and severity of symptoms related to lymphedema as assessed by the GCLQ were significantly associated with reduced QoL (Fig. 2), a finding which has been reported by others [17, 23-27]. Interestingly, the type of lymph-node dissection (sentinel LND, first line LND, or total inguinal LND) was not related to the degree of either lymphedema symptoms or QoL (Figure S2 in the supplementary online resource p.6). The latter aspect of this finding is in accord with data from a larger study by Oonk et al., reporting that there was no difference in terms of QoL between patients undergoing sentinel LND and total inguinal LND [28]. Even though there was a trend to less lymphedema in patients who received sentinel LND as compared to those who received first line or total inguinal LND, this difference was not significant, probably because of our small-sample size (Figure S3 in the online resource p. 7). It should be noted that comparison of our lymphedema findings with results from other trials is limited, because generally different assessment methods are used. Lymphedema is commonly evaluated by clinical observation, assessment of pitting, measurements (e.g., leg circumference or water displacement measurements), and bioimpedance spectroscopy. The GCLQ, on the other hand, assesses patient-reported symptoms. While patient-reported symptoms seem more relevant than clinical signs of lymphedema, GCLQ scores might also overestimate the incidence of true lymphedema. This is supported by a recent study involving lymphedema assessment in 30 vulvar cancer patients in which the investigators demonstrated that self-reported lymphedema was present in $12 / 18$ patients (67\%), while it could be detected by bioimpedance measurements in 1/12 (8.3\%) only [29]. Other studies, however, have shown that clinical findings and GCLQ scores correlate reasonably well [12, 30, 31].

Only $18.6 \%$ of our patients reported to be sexually active. This number corresponds well with the results from other studies reporting sexual activity in $17.7 \%$ [28] and $21.4 \%$ [23]. In the latter study, the investigators demonstrated that there was no difference in sexual activity in a comparable cohort of healthy patients. In a comprehensive review [32], Aerts et al. concluded that besides a history of depression and excision size of the vulvar lesion, poor overall QoL and patient age were the most important determinants of sexual activity, a finding which our study confirms (Fig. 4). Importantly, both QoL and sexual activity are significantly influenced by age. In a study by Grimm et al. reporting QoL and sexual functioning of 21 vulvar cancer patients undergoing conventional surgical treatment, $38.1 \%$ of the women were sexually active [33]. However, the median patient age in that study was 52 years as compared to 63 years in our study. Interestingly, out of 12 patients who specified the reason for their sexual inactivity, eight $(66.7 \%)$ stated physical problems related to the surgery interfering with sexual intercourse [33]. In our study, only $28.1 \%$ of patients attributed their sexual inactivity to VFR sequelae.

To our knowledge, this is the largest cohort of anatomically reconstructed patients of whom QoL outcomes are reported. As has been shown before, anatomic reconstruction is an important determinant of good functional status and cosmetic outcome [34-36]. However, up to date, there have been no prospective studies evaluating the impact of different reconstructive techniques or anatomical reconstruction in general on QoL. One recent retrospective analysis including 12 vulvar cancer cases who received reconstruction using pedicle flaps reported a mean global QoL score of 75.7 [16]. This number cannot be compared to our collective, however, because no information regarding regional treatment was available and the sample size is small. In our patients, we did not observe a difference in QoL between the different types of flaps used (Figure S4 in the supplementary online resource p. 7).

Our study has several important weaknesses. First, it is a single-group observational study and the results can only be compared to historic controls from similar cohorts preventing any inferences on causality and all analyses have to be considered exploratory. Second, the generalization of our findings is restricted by the small-sample size. This, however, is a common limitation in vulvar cancer studies as it is a rare—although increasing — entity. Third, our study design did not include preoperative QoL assessment which prohibits any definitive statement regarding the effect of VFR on QoL over time. This will be determined by the results from 
our ongoing prospective multicentric VFR study. Yet, our data demonstrate that VFR in addition to leading to excellent oncological outcomes is also associated with good postoperative QoL. For the first time, we demonstrate that postoperative wound-healing complications and the presence of preoperative morbidities are associated with significantly impaired QoL. As there is an interaction between these two factors, the presence of preoperative medical comorbidities seems to identify a patient collective both at risk for woundhealing disturbances and reduced quality of life. Further studies including our own ongoing multicentric VFR trial need to corroborate these findings.

Acknowledgements Open Access funding provided by Projekt DEAL.

Author contributions S. Trott: project development, data collection and management, data analysis, and manuscript writing. M. Höckel: project development, data collection and management, data analysis, manuscript editing, and principal investigator of the Leipzig School VFR study. N. Dornhöfer: data collection and manuscript editing. K. Geue: Project development, data analysis, and manuscript editing. B. Aktas: Data analysis, manuscript editing, and investigator of the Leipzig School VFR study. B. Wolf: Project development, data collection and management, data analysis, and manuscript writing.

Funding The study was supported by Stiftung Gynäkologische Onkologie, a non-profit foundation committed to the support of research in gynecologic oncology. The foundation had no role in any aspect of preparation, conduct, analysis, or preparation of the report of this study.

\section{Compliance with ethical standards}

Conflicts of interest B. Aktas has received personal fees from Novartis Pharma, AstraZeneca, Amgen, and Roche Pharma outside the submitted work. N. Dorhöfer has received personal fees from Roche Pharma, AstraZeneca, and Tesaro outside the submitted work. B. Wolf and M. Höckel were supported by a grant from Stiftung Gynäkologische Onkologie.

Open Access This article is licensed under a Creative Commons Attribution 4.0 International License, which permits use, sharing, adaptation, distribution and reproduction in any medium or format, as long as you give appropriate credit to the original author(s) and the source, provide a link to the Creative Commons licence, and indicate if changes were made. The images or other third party material in this article are included in the article's Creative Commons licence, unless indicated otherwise in a credit line to the material. If material is not included in the article's Creative Commons licence and your intended use is not permitted by statutory regulation or exceeds the permitted use, you will need to obtain permission directly from the copyright holder. To view a copy of this licence, visit http://creativecommons.org/licenses/by/4.0/.

\section{References}

1. Siegel RL, Miller KD (2017) Jemal A (2017) Cancer Statistics. CA Cancer J Clin 67(1):7-30. https://doi.org/10.3322/caac.21387

2. Stewart BW, Wild CP (2014) World Cancer Report 2014 International Agency for Research on Cancer. World Health Organization, Lyon
3. Holleczek B, Sehouli J, Barinoff J (2018) Vulvar cancer in Germany: increase in incidence and change in tumour biological characteristics from 1974 to 2013. Acta Oncol 57(3):324-330. https:// doi.org/10.1080/0284186X.2017.1360513

4. Meltzer-Gunnes CJ, Småstuen MC, Kristensen GB et al (2017) Vulvar carcinoma in Norway: A 50-year perspective on trends in incidence, treatment and survival. Gynecol Oncol 145(3):543548. https://doi.org/10.1016/j.ygyno.2017.03.008

5. Buttmann-Schweiger N, Klug SJ, Luyten A et al (2015) Incidence patterns and temporal trends of invasive nonmelanotic vulvar tumors in Germany 1999-2011. A population-based cancer registry analysis. PLoS ONE 10(5):e0128073. https://doi.org/10.1371/ journal.pone.0128073

6. National Cancer Institute SEER Cancer Stat Facts: Vulvar Cancer. https://seer.cancer.gov/statfacts/html/vulva.html. Accessed 23 Sep 2019

7. Höckel M, Schmidt K, Bornmann K et al (2010) Vulvar field resection: novel approach to the surgical treatment of vulvar cancer based on ontogenetic anatomy. Gynecol Oncol 119(1):106113. https://doi.org/10.1016/j.ygyno.2010.06.019

8. Höckel M, Trott S, Dornhöfer N et al (2018) Vulvar field resection based on ontogenetic cancer field theory for surgical treatment of vulvar carcinoma: a single-centre, single-group, prospective trial. Lancet Oncol 19(4):537-548. https://doi.org/10.1016/S1470 $-2045(18) 30109-8$

9. Aaronson NK, Ahmedzai S, Bergman B et al (1993) The European Organization for Research and Treatment of Cancer QLQC30: a quality-of-life instrument for use in international clinical trials in oncology. JNCI 85(5):365-376. https://doi.org/10.1093/ jnci/85.5.365

10. Preston NJ, Wilson N, Wood NJ et al (2015) Patient-reported outcome measures for use in gynaecological oncology: a systematic review. BJOG 122(5):615-622. https://doi. org/10.1111/1471-0528.13251

11. Schwarz R, Hinz A (2001) Reference data for the quality of life questionnaire EORTC QLQ-C30 in the general German population. Eur J Cancer 37(11):1345-1351

12. Carter J, Raviv L, Appollo K et al (2010) A pilot study using the Gynecologic Cancer Lymphedema Questionnaire (GCLQ) as a clinical care tool to identify lower extremity lymphedema in gynecologic cancer survivors. Gynecol Oncol 117(2):317-323. https://doi.org/10.1016/j.ygyno.2010.01.022

13. Fayers PM, Aaronson NK, Bjordal K et al (2001) The EORTC QLQ-C30 Scoring Manual, 3rd edn. European Organisation for Research and Treatment of Cancer, Brussels

14. Core Team R (2018) R: A language and environment for statistical computing. R Foundation for Statistical Computing, Vienna, Austria Available online at www.R-project.org

15. Hinz A, Singer S, Brähler E (2014) European reference values for the quality of life questionnaire EORTC QLQ-C30: results of a German investigation and a summarizing analysis of six European general population normative studies. Acta Oncol 53(7):958-965. https://doi.org/10.3109/0284186X.2013.879998

16. Hellinga J, Te Grootenhuis NC, Werker PMN et al (2018) Quality of life and sexual functioning after vulvar reconstruction with the lotus petal flap. Int J Gynecol Cancer 28(9):1728-1736. https://doi.org/10.1097/IGC.0000000000001340

17. Novackova M, Halaska MJ, Robova H et al (2015) A prospective study in the evaluation of quality of life after vulvar cancer surgery. Int J Gynecol Cancer 25(1):166-173. https://doi. org/10.1097/IGC.0000000000000313

18. Wang R, Zhao Y, He X et al (2009) Impact of hypertension on health-related quality of life in a population-based study in Shanghai. China Public Health 123(8):534-539. https://doi. org/10.1016/j.puhe.2009.06.009 
19. Rubin RR, Peyrot M (1999) Quality of life and diabetes. Metab Res Rev 15(3):205-218

20. Jones GL, Jacques RM, Thompson J et al (2016) The impact of surgery for vulval cancer upon health-related quality of life and pelvic floor outcomes during the first year of treatment: a longitudinal, mixed methods study. Psychooncology 25(6):656-662. https://doi.org/10.1002/pon.3992

21. Schwartz CE, Sprangers MAG (1999) Methodological approaches for assessing response shift in longitudinal healthrelated quality-of-life research. Soc Sci Med 48(11):1531-1548. https://doi.org/10.1016/S0277-9536(99)00047-7

22. Sprangers MAG, Schwartz CE (1999) Integrating response shift into health-related quality of life research: a theoretical model. Soc Sci Med 48(11):1507-1515. https://doi.org/10.1016/S0277 -9536(99)00045-3

23. de MeloFerreira AP, de Figueiredo EM, Lima RA et al (2012) Quality of life in women with vulvar cancer submitted to surgical treatment: a comparative study. Eur J Obstet Gynecol Reprod Biol 165(1):91-95. https://doi.org/10.1016/j.ejogr b.2012.06.027

24. Novackova M, Halaska MJ, Robova H et al (2012) A prospective study in detection of lower-limb lymphedema and evaluation of quality of life after vulvar cancer surgery. Int J Gynecol Cancer 22(6):1081-1088. https://doi.org/10.1097/IGC.0b013e31825866d 0

25. Farrell R, Gebski V, Hacker NF (2014) Quality of life after complete lymphadenectomy for vulvar cancer: do women prefer sentinel lymph node biopsy? Int J Gynecol Cancer 24(4):813-819. https://doi.org/10.1097/IGC.0000000000000101

26. Janda M, Obermair A, Cella D et al (2004) Vulvar cancer patients' quality of life: a qualitative assessment. Int J Gynecol Cancer 14(5):875-881. https://doi.org/10.1111/j.1048-891X.2004.14524 . $\mathrm{x}$

27. Kim SI, Lim MC, Lee JS et al (2015) Impact of lower limb lymphedema on quality of life in gynecologic cancer survivors after pelvic lymph node dissection. Eur J Obstet Gynecol Reprod Biol 192:31-36. https://doi.org/10.1016/j.ejogrb.2015.06.011

28. Oonk MHM, van Os MA, de Bock GH et al (2009) A comparison of quality of life between vulvar cancer patients after sentinel lymph node procedure only and inguinofemoral lymphadenectomy. Gynecol Oncol 113(3):301-305. https://doi.org/10.1016/j. ygyno.2008.12.006
29. Hayes SC, Janda M, Ward LC et al (2017) Lymphedema following gynecological cancer: Results from a prospective, longitudinal cohort study on prevalence, incidence and risk factors. Gynecol Oncol 146(3):623-629. https://doi.org/10.1016/j.ygyno .2017.06.004

30. Do JH, Choi KH, Ahn JS et al (2017) Effects of a complex rehabilitation program on edema status, physical function, and quality of life in lower-limb lymphedema after gynecological cancer surgery. Gynecol Oncol 147(2):450-455. https://doi.org/10.1016/j. ygyno.2017.09.003

31. Lim MC, Lee JS, Joo J et al (2014) Development and evaluation of the Korean version of the Gynecologic Cancer Lymphedema Questionnaire in gynecologic cancer survivors. Gynecol Oncol 133(1):111-116. https://doi.org/10.1016/j.ygyno.2014.01.040

32. Aerts L, Enzlin P, Vergote I et al (2012) Sexual, psychological, and relational functioning in women after surgical treatment for vulvar malignancy: a literature review. J Sex Med 9(2):361-371. https://doi.org/10.1111/j.1743-6109.2011.02520.x

33. Grimm D, Hasenburg A, Eulenburg C et al (2015) Sexual Activity and function in patients with gynecological malignancies after completed treatment. Int J Gynecol Cancer 25(6):1134-1141. https://doi.org/10.1097/IGC.0000000000000468

34. Zhang W, Zeng A, Yang J et al (2015) Outcome of vulvar reconstruction in patients with advanced and recurrent vulvar malignancies. BMC Cancer 15:851. https://doi.org/10.1186/s1288 5-015-1792-x

35. Höckel M, Dornhöfer N (2008) Vulvovaginal reconstruction for neoplastic disease. Lancet Oncol 9(6):559-568. https://doi. org/10.1016/S1470-2045(08)70147-5

36. Di Donato V, Bracchi C, Cigna E et al (2017) Vulvo-vaginal reconstruction after radical excision for treatment of vulvar cancer: Evaluation of feasibility and morbidity of different surgical techniques. Surg Oncol 26(4):511-521. https://doi.org/10.1016/j. suronc.2017.10.002

Publisher's Note Springer Nature remains neutral with regard to jurisdictional claims in published maps and institutional affiliations. 